\title{
Automatic and strategic effects in semantic priming: An examination of Becker's verification model
}

\author{
KEN den HEYER \\ St. Francis Xavier University, Antigonish, Nova Scotia, Canada \\ and \\ KEVIN BRIAND and LEE SMITH \\ Dalhousie University, Halifax, Nova Scotia, Canada
}

\begin{abstract}
Becker's (1980) verification model, in conjunction with a two-strategy hypothesis, is cited as an alternative to a dual-process model (e.g., Posner \& Snyder, 1975) of word recognition and semantic priming. Becker's approach suggests that individuals can use either an "expectancy" or a "prediction" strategy in word recognition, and maintains that the verification model successfully predicts certain patterns of facilitation and inhibition in a semantic priming task that a dual-process model cannot. The present study demonstrated that when a long stimulus-onset asynchrony ( $\mathrm{SOA}=1,000 \mathrm{msec}$ ) is used between prime and target, support is given to Becker's findings, and to the verification model approach. However, at a short SOA (200 msec), no evidence is found for the hypothesized difference between strategic processes. The results are consistent with other findings (e.g., Neely, 1977) in showing that strategic factors in semantic priming are largely inoperative at short prime-target SOAs, and suggests that Becker's model is not general enough to rule out some type of dual-process model.
\end{abstract}

An increasing number of studies have in the last few years been directed at the nature of semantic priming (e.g., Antos, 1979; Becker, 1979, 1980; de Groot, Thomassen, \& Hudson, 1982; den Heyer, Briand, \& Dannenbring, 1983; Fischler, 1977a, 1977b; Lorch, 1982; Meyer \& Schvaneveldt, 1971; Meyer, Schvaneveldt, \& Ruddy, 1975; Neely, 1976, 1977; Stanovich \& West, $1979,1981)$. The central finding in this research is that the processing of a word or target is facilitated if that word or target is preceded by a related word or prime. Although there are a large number of methodological variations, a common procedure involves a lexical decision task (word-nonword decision).

Following the dual-process model of Posner and Snyder (1975), much of the current research concerned with semantic priming is aimed at automatic and attentioninduced priming effects. Attention-induced priming is distinguished from automatic priming in that the former requires effort and processing capacity and is relatively

This research was supported by a research grant from the Natural Sciences and Engineering Research Council of Canada to the first author. Part of this research was completed while the first author was on leave at Acadia University. The results of the present research were presented at the annual convention of the Canadian Psychological Association, June 1984, Ottawa. The authors would like to express their gratitude for the very detailed and useful comments by one anonymous reviewer. Requests for reprints should be addressed to Ken den Heyer, Department of Psychology, St. Francis Xavier University, Antigonish, N.S., Canada B2G $1 \mathrm{CO}$. slow, whereas the latter requires no effort or processing capacity and is rapid. Furthermore, attention-induced priming is associated with inhibitory effects for unprimed nodes, whereas no such inhibitory effects are associated with automatic priming. Much of the support for this approach comes from the work by Neely (1977). Using a procedure in which subjects were given a category name as a prime and a target from another category, Neely found a priming effect when such a pattern was expected and when the interval between prime and target presentation (SOA) was substantial. Furthermore, Neely also reported inhibition for unrelated prime-target pairs. However, at a shorter SOA, the inhibitory and expectancy-induced priming effects disappeared and left only a priming effect for the targets "naturally" or semantically related to the primes, indicating that only automatic priming can occur when the SOA is brief. Recently, a set of findings similar to those of Neely were reported by Favreau and Segalowitz (1983).

In addition to the work by Neely (1977), a number of studies have varied the proportion of related trials in a semantic priming procedure (de Groot, 1984; den Heyer et al., 1983; Koriat, 1981; Simpson \& Lorsbach, 1983; Tweedy \& Lapinski, 1981; Tweedy, Lapinski, \& Schvaneveldt, 1977). All of these studies have reported that amount of facilitation or amount of priming correlated positively with the proportion of related word-word pairs, indicating some form of attention-induced priming. Also, den Heyer et al. (1983) reported that the proportion ef- 
fect disappeared when the SOA was short, lending further support to the distinction between attention-induced and automatic priming effects.

Recently, Becker (1980) reported a pattern of priming results that runs counter to the Posner and Snyder (1975) dual-process account of priming effects. Becker reported a substantial priming effect for related pairs and an inconsequential inhibitory effect for unrelated pairs as measured against a neutral priming condition in which the stimuli were composed of antonyms. When the primetarget pairs took the form of category exemplars, however, little facilitation for related pairs and substantial inhibition for unrelated pairs were found. As Becker pointed out, the correlation between inhibition and facilitation should be positive-not negative-if attentioninduced priming effects follow the Posner and Snyder dual-process model. In this context, it should be noted that West, Stanovich, Freeman, and Cunningham (1983) provided correlations between amount of facilitation and inhibition across subjects and failed to find any significant relationships. However, West et al. used a sentence rather than a word priming procedure.

Becker (1980) accounts for his results by reference to a verification model of word recognition (Becker, 1976, 1979, 1980; Becker \& Killion, 1977). Very briefly, this model holds that a prime establishes a semantic candidate set of word items. On presentation of a target, the sensory representation of the target is first checked sequentially against the semantic candidate set. If no match occurs, as would be the case for the unrelated priming condition, then the sensory representation of the target is verified against a sensory candidate set derived from the sensory representation of the target. Becker maintains that, in the case of antonym stimuli, a prime elicits only a small semantic candidate set, since subjects develop a prediction strategy consisting of predicting likely opposites to the prime. For category-exemplar stimulus materials, however, the semantic candidate sets will be relatively large, since a large number of alternatives can be expected. The neutral priming condition does not involve a semantic candidate set. Here, target processing proceeds by verifying the sensory representation of the target against the sensory candidate set after the sensory candidate set is established. In effect, the model suggests that response latency for both related and unrelated priming conditions correlates positively with semantic candidate set size, whereas the neutral priming condition remains unaffected. Thus, when primes elicit large semantic candidate sets, the patterns of results are inhibition dominant, and conversely, when primes typically elicit small candidate sets, the results are facilitation dominant.

A problem with the verification model is that it has not been applied to different time relations between prime and target presentation. A strength of the dual-process model is that it successfully predicts the interaction between prime-target SOA and context with respect to attentioninduced priming effects. This problem has been recognized (Eisenberg \& Becker, 1982), but not addressed.

In effect, the purpose of this study is twofold. First, we intend to replicate the Becker (1980) results with respect to the antonym-antonym and category-exemplar stimulus sets. Although we have no reason to doubt Becker's data, we are not aware of any reports that have replicated those results. Since Becker's findings have important theoretical consequences for models of semantic priming and, more generally, for models of word recognition, confirmation of these results with independently selected stimulus sets and subject samples appears to be in order. The second intention of this study is to examine the differences between the above-mentioned stimulus sets as a function of prime-target SOA. If the difference between the inhibition- and facilitation-dominant stimulus sets breaks down and, more importantly, if inhibition disappears, then a limit has been placed on the generality of the verification model.

\section{METHOD}

\section{Subjects}

A total of 120 individuals, from the subject pool at St. Francis Xavier University, participated in this experiment. All subjects were paid for their participation.

\section{Procedure}

All subjects were run individually in a single session that lasted approximately $30 \mathrm{~min}$. Stimuli were presented on a computercontrolled video display. The screen was about 18 in away from the subjects, at approximately eye level. Two keys were used for the WORD and NONWORD responses; the preferred hand was always used for the WORD response decision.

The 120 subjects were divided into four equal groups. Two different stimulus sets (see below) and two different SOAs of 200 and $1.000 \mathrm{msec}$ were used.

The subjects were told that they would be shown letter strings on the screen, and that they would have to decide whether or not the strings were words. They were told to make their responses to the target items as quickly as possible. On each trial, the sequence of events was as follows. First, a warning cue (a row of five asterisks) appeared for about $1 \mathrm{sec}$. Next, the prime appeared below the warning signal. Either 200 or $1,000 \mathrm{msec}$ after presentation of the prime, the target appeared below the prime; both the target and the prime remained in view until the subject had responded. The subjects were told that the prime item would be either a word or a string of five "Xs," and that this would be followed by the target, which would be either a word or a nonword. After the subject had made his or her response, the screen was cleared, and an intertrial interval of approximately $4 \mathrm{sec}$ followed before the next trial began. Trials on which the subjects responded incorrectly were replaced in the pool of yet-to-be-presented trials.

Before the experimental session, each of the subjects received 50 practice trials on materials similar to, but not the same as, items used for the experimental trials. Furthermore, the subjects were carefully apprised of the nature of the stimuli and of the relationships between prime and target. ${ }^{1}$ During the session, rest periods were given after every 30 trials; the subjects controlled the duration of these rest periods by pressing a button on the keyboard when they were ready to resume the experiment.

\section{Stimuli}

The stimuli were chosen in such a way as to replicate Becker's (1980, Experiments $t$ and 2) procedure. The antonym and category sets will be described separately.

\footnotetext{
Antonym Set

A total of 30 pairs of antonyms (e.g.. GIVE-TAKE) were chosen and matched so that the frequencies of occurrence of prime and
} 
target were as equal as possible on the basis of the norms of Kučera and Francis (1967). The average frequency for the prime words was 400.4 , and that of the targets was 296.0 . For each subject, the set of 30 pairs was divided randomly into three sets of 10 pairs each. One of these sets used the given primes and targets as a critical set of related stimuli. For a second set of 10 , the given prime words were used, but the targets were chosen from the total of 20 remaining targets, so that primes and targets formed a critical set of 10 unrelated stimuli. For the 10 remaining primes and targets, the primes were replaced by neutral stimuli (XXXXX), and the targets were included unchanged. Thus, the critical stimuli consisted of sets of 10 related word pairs, 10 unreiated word pairs, and 10 neutral-prime word pairs, with no overlap between sets for a given subject.

In addition to the above critical items, filler materials were used. A second related word (e.g., THIN-FAT) had been chosen for each of the original prime stimuli. Thirty additional related-filler trials were structured by presenting those targets with the appropriate cue. The 10 cue words that had been replaced by neutral primes for the critical trials were paired with 10 unrelated filler words. Thus, as with Becker's (1980) procedure, all primes were shown twice and then followed by a target requiring a word response. Thirty additional filler words served as targets for neutral primes, resulting in 100 trials with word targets. Fifty nonwords were structured by changing one or two letters in words chosen from the same source as the critical stimuli, and an effort was made to preserve the original sound of the word (as did Becker, 1980). These were used as targets and were preceded by the 30 critical prime words and 20 neutral primes. The breakdown of the 150 trials in the antonym set, listed in Table 1 , replicated the proportions used by Becker.

\section{Category Set}

Critical materials were chosen from Battig and Montague (1969). A total of 18 category names were used as primes that could be represented by a single word. For each category name, three members were chosen from that category for use as possible target stimuli. These corresponded to high-, moderate-, and low-typicality members, with the constraint that no target stimulus have a Battig and Montague frequency of occurrence of less than 10 . The average Battig and Montague response counts for the resulting high-, moderate-, and low-typicality targets were $353.3,155.8$, and 42.2 , respectively. Attempts were made to choose these target stimuli in such a way that they were as close as possible in word frequency (Kučera \& Francis, 1967). The corresponding frequency counts for the above stimuli were $46.8,24.4$, and 39.1 .

Once again, stimulus materials were selected in accordance with the procedure used by Becker (1980). First of all, six high-typicality items were chosen randomly to follow the appropriate category names for six critical related pairs. Six more high-typicality items were chosen randomly to follow neutral primes (the category name was replaced as a prime), and, in order to create six unrelated trials, the remaining six had the appropriate category names replaced by those category names not used in the neutral trials. The assignments of stimulus materials for the moderate- and low-typicality targets were counterbalanced. The same three sets of categories used to generate the related, unrelated, and neutral trials from the hightypicality targets were maintained for the low-typicality targets, but the three different prime sets were assigned to different prime-target conditions. The resultant set of 54 critical stimulus pairs consisted of 18 related, 18 unrelated, and 18 neutral stimulus pairs. Each set of 18 consisted of six items of one of three levels of typicality, and the three category exemplars were always used in three different prime-type conditions (i.e., related, unrelated, and neutral).

Thus, as in Becker's (1980) procedure, each category cue was presented twice, once followed by a related category exempiar and once followed by an unrelated category exemplar. No target items were repeated in the critical item set of 54 stimulus pairs.

In addition to the critical stimulus pairs, filler materials were generated. Nine other category names were chosen, and two high-
Table 1

Summary of the Design for the Antonym and Category Stimulus Sets

\begin{tabular}{lll}
\hline \multicolumn{1}{c}{ Condition } & \multicolumn{1}{c}{ Example } & Number of Trials \\
\hline Word-Word Related & \multicolumn{1}{c}{ Antonym Set } & \\
Word-Word Unrelated & FIRST-LEFT & 10 \\
Neutral-Word & XXXXX-STRONG & 10 \\
Related Fillers & QUIET-HOUSE & 10 \\
Unrelated Fillers & FAST-THEY & 30 \\
Neutral Fillers & XXXXX-CLEAR & 30 \\
Word-Nonword & GOOD-BLOO & 30 \\
Neutral-Nonword & XXXXX-WIRKS & 20 \\
& $\quad$ Category Set & \\
Word-Word Related & METAL-COPPER & 18 \\
Word-Word Unrelated & WEAPON-COAT & 18 \\
Neutral-word & XXXXX-LINEN & 18 \\
Related Fillers & SPORT-BOXING & 18 \\
Neutral Fillers & XXXXX-SPARROW & 18 \\
Word-Nonword & Fruit-SNOE & 27 \\
Neutral-Nonword & XXXXX-BRANE & 18 \\
\hline
\end{tabular}

and two low-typicality category exemplars were found for each. The nine category names were paired twice, once with a lowtypicality and once with a high-typicality category exemplar. The remaining 18 filler words were used as targets preceded by a neutral prime. Forty-five nonwords were generated (as in the antonym set) and used as targets. Eighteen were preceded by neutral primes, and the remainder by the 27 category names $(18$ critical ones and 9 fillers). A summary of the various kinds of priming trials is listed in Table 1.

\section{RESULTS}

The median reaction times for the various experimental conditions for each subject were calculated and formed the basis for further data analysis. Figure 1 summarizes the reaction times for the word data and for the error data.

A three-factor (stimulus set, SOA, and prime-type) analysis of variance was applied to the reaction time data for the "WORD" responses for the critical target sets. The analysis produced a significant priming effect $[F(2,232)$ $=24.38, \mathrm{p}<.001]$. Neither the main effect of SOA nor that of stimulus set was significant at the $5 \%$ level. The interaction between SOA and priming condition was significant $[F(2,232)=4.69, \mathrm{p}<.05]$, reflecting a smaller prime-type effect at the shorter SOA. The interaction between stimulus set and priming condition was significant, as was the second-order interaction between stimulus set, SOA, and priming condition $[\mathrm{F}(2,232)=4.11, \mathrm{p}<.05$, and $F(2,232)=3.26, p<.05$, respectively].

The word reaction times were also analyzed for each SOA condition. The $1,000-\mathrm{msec}$ SOA condition produced a significant priming effect $[F(2,116)=22.76, p<.001]$ and a significant stimulus set $\times$ prime type interaction effect $[F(2,116)=6.58, p<.01]$. Inspection of Figure 1 indicates that the significant interaction is due to the fact that the antonym stimulus set produced a facilitationdominant pattern of results, whereas the category stimuli produced an inhibition-dominant pattern of response times. A similar analysis of the 200 -msec data produced a significant priming effect $[F(2,116)=5.62, p<.01]$. 


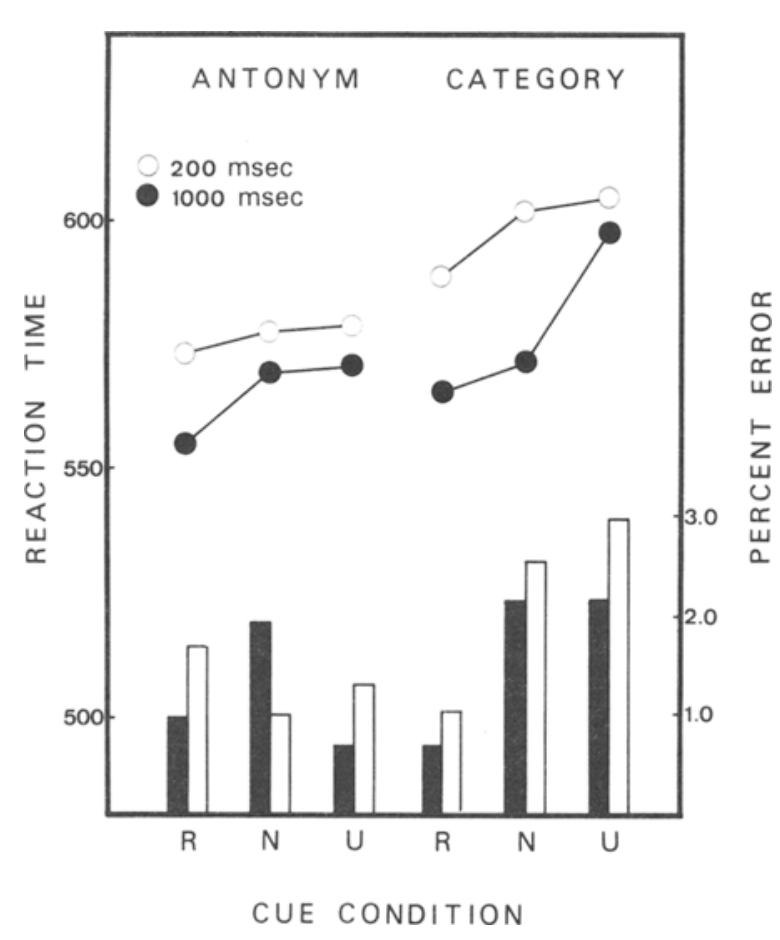

Figure 1. Average reaction times and percent error as a function of stimulus set, SOA, and priming condition.

The interaction between stimulus set and priming condition was not significant $[\mathrm{F}(2,116)=1.34, \mathrm{p}>.25]$, reflecting the fact that priming was facilitation dominant for both types of stimulus materials.

A three-factor analysis of the error data yielded a significant interaction between stimulus set and prime type $[F(2,232)=3.16, p<.05]$. No other effects were significant. A separate analysis for each of the two SOA conditions did not produce any significant effects.

The typicality data were also analyzed and produced a typicality effect $[F(2,116)=33.79, p<.001]$. Typicality did not interact with either SOA or prime type.

\section{DISCUSSION}

This study was intended to address the argument raised by Becker (e.g., Becker, 1980; Eisenberg \& Becker, 1982) that his verification model, in conjunction with two hypothesized strategies of word recognition, represents a viable alternative to dual-process-based models of word recognition and semantic priming. With different stimuli-or at least independently selected stimuli-and more subjects ( 60 vs. 48 ), the pattern of results indicated a qualitative difference in performance on the two stimulus sets. Futhermore, the patterns of facilitation and inhibition dominance demonstrated at an SOA of $1,000 \mathrm{msec}$ between the presentation of the prime and target did not occur at a shorter SOA. Performance of subjects on the two stimulus sets at the shorter SOA yielded essentially identical results, with both sets producing a facilitation-dominant priming pattern. As Becker himself suggested (Eisenberg \& Becker, 1982), the model he proposed does not apply when the SOA between prime and target presentation is short. In effect, the short-SOA results support Becker's interpretation of the stimulus set effect's being strategic in nature. At the same time, these results also support the automatic-strategic distinction proposed by Posner and Snyder (1975).

The problem that must now be resolved is the supposed incompatibility between the two models. Becker (1980) was quite clear on this issue. He stated that the dualprocess model predicts a positive correlation between inhibition and facilitation if differences in amount of priming are a function of differing degrees of attention-induced facilitation. There are basically two directions in which this incompatibility may be resolved. First, Becker's model can be modified or extended. It may be possible that establishment of the semantic set is time dependent, such that, at a short SOA, differences in semantic set size are minimal by the time the target arrives. In effect, this would reduce Becker's formulation to one that is analogous to automatic spreading activation when the SOA between prime and target is short.

The other possibility with respect to the incompatibility between the verification and dual-process model is to extend the dual-process model to incorporate a verification process along the lines suggested by Becker (1980). Rather than supposing that the stimulus set effect is incompatible with the dual-process model, it can be seen as extending the dual-process model. If it is assumed that the two stimulus sets do induce different strategies, it may not be appropriate to use the negative correlation between facilitation and inhibition as disconfirming evidence for the nature of attention-driven effects as promulgated by the dual-process model. In other words, different strategies may produce different patterns of results. It may still be true that, within a given strategy, the cost-benefit assumption of the dual-process model applies. Thus, for example, if proportion of related trials is manipulated within the expectancy strategy or within a prediction strategy, it is possible that this would produce results in accordance with the dual-process model, namely, larger costs and benefits as the proportion of related trials increases.

At the moment, we tend to favor the second of the two above-mentioned possibilities for two different reasons. First, the dual-process model does describe changes in performance over SOA, whereas the verification model does not. Second, manipulation of proportion of related pairs within a semantic priming procedure also appears to conform to the dual-process model (e.g., de Groot, 1984; den Heyer et al., 1983; Tweedy et al., 1977). An experiment by Becker (1980, Experiment 5) demonstrated that the prediction strategy is readily induced by including a mixture of pairs from the antonym and category lists. Thus, the proportion effect obtained by the abovementioned authors likely involves the deployment of a prediction strategy. What remains to be determined is whether or not changes in proportion of related pairs produce similar results when subjects are induced to adopt an expectancy or inhibition-dominant strategy. If this is 
indeed the case, it would represent strong support for the present interpretation.

Although the incorporation of expectancy and prediction strategies into the dual-process model may be possible, other research seems to indicate that the dual-process model may be in need of alteration. The distinction between automatic and controlled processes has become somewhat clouded. Antos (1979) and de Groot et al. (1982) have suggested that inhibition in a semantic priming paradigm may in fact be possible at short SOAs, whereas more recent work (Kahneman \& Chajczyk, 1983; Kahneman, Treisman, \& Burkell, 1983) indicates that supposedly automatic processes require processing capacity. However, neither of these two sets of results is inconsistent with the notion that cognitive control over processing is time dependent and that attention-controlled processes are generally ineffective at short prime-target SOAs.

\section{REFERENCES}

Antos, S. J. (1979). Processing facilitation in a lexical decision task. Journal of Experimental Psychology: Human Perception and Performance, 5, 527-545.

Battig, W. F., \& Montague, W. E. (1969). Category norms for verbal items in 56 categories: A replication and extension of the Connecticut category norms. Journal of Experimental Psychology Monographs, 80(3, Pt.2).

BECKER, C. A. (1976), Allocation of attention during visual word recognition. Journal of Experimental Psychology: Human Perception and Performance, 2, 555-566.

BECKER, C. A. (1979). Semantic context and word frequency effects in visual word recognition. Journal of Experimental Psychology: Human Perception and Performance, 5, 252-259.

BECKER, C. A. (1980). Semantic context effects in visual word recognition: An analysis of semantic strategies. Memory \& Cognition, 8, 493-512.

BeCKer, C. A., \& Killion, T. H. (1977). Interaction of visual word recognition. Joumal of Experimental Psychology: Human Perception and Performance, 3, 389-401.

DE GRoot, A. M. B. (1984). Primed lexical decision: Combined effects of the proportion of related prime-target pairs and the stimulusonset-asynchrony of prime and target. Quarterly Journal of Experimental Psychology, 36A, 253-280.

de Groot, A. M. B., Thomassen, A. J. W. M., \& Hudson, P. T. W. (1982). Associative facilitation of word recognition as measured from a neutral prime. Memory \& Cognition, 10, 358-370.

den Heyer, K., Briand, K., \& Dannenbring, G. L. (1983). Strategic factors in a lexical-decision task: Evidence for automatic and attention-driven processes. Memory \& Cognition, 11, 374-381.

EISENBERG, P., \& BECKER, C. A. (1982). Semantic context effects in visual word recognition, sentence processing, and reading: Evidence for semantic strategies. Journal of Experimental Psychology: Human Perception and Performance, 8, 739-756.

Favreau, M., \& Segalowitz, N. S. (1983). Automatic and controlled processes in the first- and second-language reading of fluent bilinguals. Memory \& Cognition, 11, 565-574.

FISCHLER, I. (1977a). Associative facilitation without expectancy in a lexical decision task. Journal of Experimental Psychology: Human Perception and Performance, 3, 18-26.

FISCHLER, I. (1977b). Semantic facilitation without association in a lexical decision task. Memory \& Cognition, 5, 335-339.

Fischler, I., \& GoOdman, G. O. (1978). Latency of associative activation in memory. Journal of Experimental Psychology: Human Perception and Performance, 4, 455-470.

KahNeman, D., \& CHAICZYK, D. (1983). Tests of the automaticity of reading: Dilution of Stroop effects by color-irrelevant stimuli. Jour- nal of Experimental Psychology: Human Perception and Performance, 9, 497-509.

Kahneman, D., Treisman, A., \& Burkell, J. (1983). The cost of visual filtering. Joumal of Experimental Psychology: Human Perception and Performance, 9, 510-522.

Koriat, A. (1981). Semantic facilitation in lexical decision as a function of prime-target association. Memory \& Cognition, 9, 587-598.

KuČera, H., \& Francis, W. N. (1967), Computational analysis of present-day American English. Providence, RI: Brown University Press.

LORCH, R. F. (1982). Priming and search processes in semantic memory: A test of three models of spreading activation. Journal of Verbal Learming and Verbal Behavior, 21, 468-492.

Meyer, D. E., \& Schvaneveldt, R. W. (1971). Facilitation in recognizing pairs of words: Evidence of a dependence between retrieval operations. Journal of Experimental Psychology: General, 90, 227-234.

Meyer, D. E., Schyaneveldt, R. S., \& Ruddy, M. G. (1975). Loci of contextual effects on visual word recognition. In P. M. A. Rabbitt \& S. Dornic (Eds.), Attention and performance V. New York: Academic Press.

NeELY, J. H. (1976). Semantic priming and retrieval from lexical memory: Evidence for facilitatory and inhibitory processes. Memory \& Cognition, 4, 648-654.

NeELY, J. H. (1977). Semantic priming and retrieval from lexical memory: Role of inhibitionless spreading activation and limitedcapacity attention. Journal of Experimental Psychology: General, 106, 226-254.

PosNer, M. I. \& SNYder, C. R. R. (1975). Facilitation and inhibition in the processing of signals. In P. M. A. Rabbitt \& S. Dornic (Eds.), Attention and performance $V$. New York: Academic Press.

Simpson, G. B., \& Lorsbach, T. C. (1983). The development of automatic and conscious components of contextual facilitation. Child Development, 54, 760-772.

Stanovich, K. E., \& WEST, R. F. (1979). Mechanisms of sentence context effects in reading: Automatic activation and conscious attention. Memory \& Cognition, 7, 77-85.

STANOVICh, K. E., \& WEST, R. F. (1981). The effects of sentence context on ongoing word recognition: Tests of a two-process theory. Journal of Experimental Psychology: Human Perception and Performance, 7, 658-672.

TWEEDY, J. R., \& LAPINSKI, R. H. (1981). Facilitating word recognition: Evidence for strategic and automatic factors. Quarterly Journal of Experimental Psychology, 33A, 51-59.

Tweedy, J. R., Lapinski, R. H., \& Schvaneveldi, R. W. (1977). Semantic-context effects on word recognition: Influence of varying the proportion of items presented in an appropriate context. Memory \& Cognition, 5, 84-89.

West, R. F., Stanovich, K. E., Feeman, D. J., \& Cunningham, A. E. (1983). The effect of sentence context on word recognition in second- and sixth-grade children. Reading Research Quarterly, 19 , 6-15

\section{NOTE}

1. It should be noted that the instructions to the subjects may be of critical importance. The experiment reported in this article represents a replication of an earlier effort with 34 subjects per group. In that procedure, subjects were given 10-20 practice trials and were not told of the nature of the stimuli and were not instructed about the different primetarget relationships. The results of that study failed to replicate the results of Experiments 1 and 2 reported by Becker (1980). In particular, the category data for the 1,000 -msec-SOA condition did not conform to an inhibition-dominant priming pattern, although it should also be noted that the error data did produce an inhibition-dominant pattern, suggesting that there may have been a speed-accuracy trade-off at work. It is possible that the change in instructions and the changing patterns of error rates are not independent.

(Manuscript received August 27, 1984; revision accepted for publication February 25, 1985.) 